\title{
MECHANISM OF POST-STIMULATORY ATRIAL FLUTTER IN THE DOG
}

\author{
BY \\ P. L. SHARMA* \\ From the Department of Pharmacology, St. Mary's Hospital Medical School, London W.2 \\ Received January 28, 1963
}

The mechanism of post-stimulatory atrial flutter has been extensively studied during the past 50 years. Rothberger and Winterberg (1914) believed that it was due to a rapidly discharging ectopic focus, while Lewis (1920) and Lewis, Feil, and Stroud (1920) ascribed it to a circus movement mechanism around the orifices of the two venæ cavæ with the impulse circulating in a clockwise or anticlockwise direction. Lewis, Drury, and Iliescu (1921b) also studied clinical atrial flutter and concluded that the circus movement mechanism was fully applicable in these cases.

Recently, Prinzmetal et al. $(1951,1953)$ have studied the mechanism of post-stimulatory atrial flutter in the dog and spontaneous atrial flutter in man by direct atrial leads and by high-speed films. They found no evidence of circus movement, and concluded that this arrhythmia was due to a rapidly discharging ectopic focus.

The experiments to be reported here were, therefore, designed to gain a further insight into this controversial problem.

\section{METHODS}

Mongrel dogs of either sex, weighing between 10-18 kg., were anæsthetized by intravenous administration of $25 \mathrm{mg}$. $/ \mathrm{kg}$. pentobarbitone sodium. Under positive pressure artificial respiration, the chest was opened by a midline incision and by splitting the sternum longitudinally. The pericardium was incised and the heart was cradled by stitching the pericardial flaps to the chest wall. Atrial flutter was produced by the injury-stimulation procedure of Rosenblueth and Garcia Ramos (1947). The inter-venæ-caval bridge was crushed with a stout hæmostat, and the intact area of the right atrium surrounding the injured area was stimulated electrically (square wave pulses of $1 \mathrm{msec}$. duration; frequency 20 per sec., 20 volts), for not more than 15 seconds at a time.

Direct atrial leads from the sinus node and right and left atrial appendages, and unipolar and bipolar limb leads were recorded on a Siemen-Ediswan 4-channel oscillograph. The paper speed used was $30 \mathrm{~mm}$. per sec., but in some cases the speed was doubled to allow for an accurate measurement of time intervals.

For the purpose of vagal stimulation, the right vagus nerve was divided in the neck and the peripheral stump was stimulated electrically (square wave pulses of $0.5 \mathrm{msec}$. duration; frequency 10 per sec., $10-12$ volts), for not more than 15 seconds at a time.

\section{RESULTS}

The injury-stimulation procedure usually resulted in atrial flutter with 2:1 atrio-ventricular block. The arrhythmia persisted for several hours in control experiments.

* I.C.I. Research Fellow, University of London. 
Time of Activation in Both Atria. The electrodes on the right atrial and left atrial appendages were so adjusted that sinus impulses (intrinsic deflections) were recorded simultaneously at both the electrodes. When atrial flutter was produced ( 232 episodes in $34 \mathrm{dogs}$ ), the atria were activated in sequence. In 136 episodes the right atrial appendage was activated earlier than the left atrial appendage; but in the other 96 episodes the reverse was true. The occurrence of these two patterns of sequential activation was random in any given animal and could not be determined in advance. The time lag between the recording of intrinsic deflections at the two electrodes varied between $0.016-0.024 \mathrm{sec}$. in different animals, but was remarkably constant in the same animal even when reversal of sequential activation occurred in consecutive episodes of flutter (Fig. 1). This observation can be easily explained on the basis of the circus movement theory (Fig. 3), since the excitation wave can travel in the circuit in a clockwise or anticlockwise direction.

Configuration of Flutter Waves in Limb Leads. Corresponding to the reversal of sequential activation in atrial leads, the limb leads showed reversal of flutter waves (Fig. 1). When the right atrium was activated earlier than the left atrium, the flutter waves were positive in leads I, II, III, and VF, and negative in leads VR and VL. This pattern is similar to that obtained during sinus rhythm but the amplitude and contour of flutter waves were different from those of sinus beats. When the reversal occurred in atrial leads in a subsequent episode of flutter in the same animal, the limb leads also shared in the reversal process. The flutter waves were now negative in leads II, III, and VF, and positive in leads I, VR, and VL.

Time of Activation Around the Hypothetical Circus Pathway. The time course of activation of a single flutter beat at four points on the circus pathway was studied in five dogs. The electrode positions are depicted in Fig. 2, together with the electrocardiographic records of a typical experiment. During normal sinus rhythm, the impulse spread was radial, electrodes 2 and 3 were activated at the same time and electrode 4 last of all. The first episode of flutter showed a clockwise spread of the impulse (excerpt B), and a subsequent episode showed an anticlockwise spread (excerpt C). The flutter cycle $\left(\mathrm{P}^{\prime}-\mathrm{P}^{\prime}\right.$ interval $)$ calculated from the atrial rate was $0.134 \mathrm{sec}$. This was equal to the time taken by the impulse to complete one circuit, as calculated from intrinsic deflections at the four electrodes (see arrows in excerpts B and C).

Effect of Cholinergic Factors on Atrial Flutter. Stimulation of the vagus nerve (20 observations in 8 dogs) or acetylcholine $0.5-1.0 \mathrm{mg}$. injected intravenously (46 observations in 10 dogs), converted flutter into fibrillation. When the cholinergic influence disappeared the arrhythmia terminated abruptly in most of the experiments $(81 \%)$; but in the remainder flutter at the control rate returned. Atrial flutter could be reinduced in all the experiments by electrical stimulation.

\section{Discussion}

Lewis $(1920,1925)$ in his classical studies advanced the circus movement theory to explain the mechanism of post-stimulatory atrial flutter. He concluded from his animal experiments that the impulse travelled in a circular manner around the openings of venæ cavæ. A criticism of the circus movement theory is that the returning cycle of the circus wave has never been demonstrated experimentally. Lewis assumed the existence of the returning cycle purely on theoretical grounds. Also, Prinzmetal et al. $(1951,1953)$ studied the mechanism of atrial flutter by recording direct atrial leads and by high-speed films, but did not find any evidence of circus movement: they concluded that the arrhythmia behaved like an ectopic focal discharge. One suitable method to distinguish ectopic focal discharge from circus movement would be to time the impulse propagation simultaneously on both the anterior and posterior surfaces of the right atrium. If flutter is due to an ectopic focus, the impulse would spread in a radial fashion in all available directions. A circus movement on the other hand would show unidirectional conduction of impulses.

In the present study, the placement of an electrode on the posterior surface of the right atrium, i.e. on the posterior segment of a hypothetical circus pathway, has demonstrated clearly the 


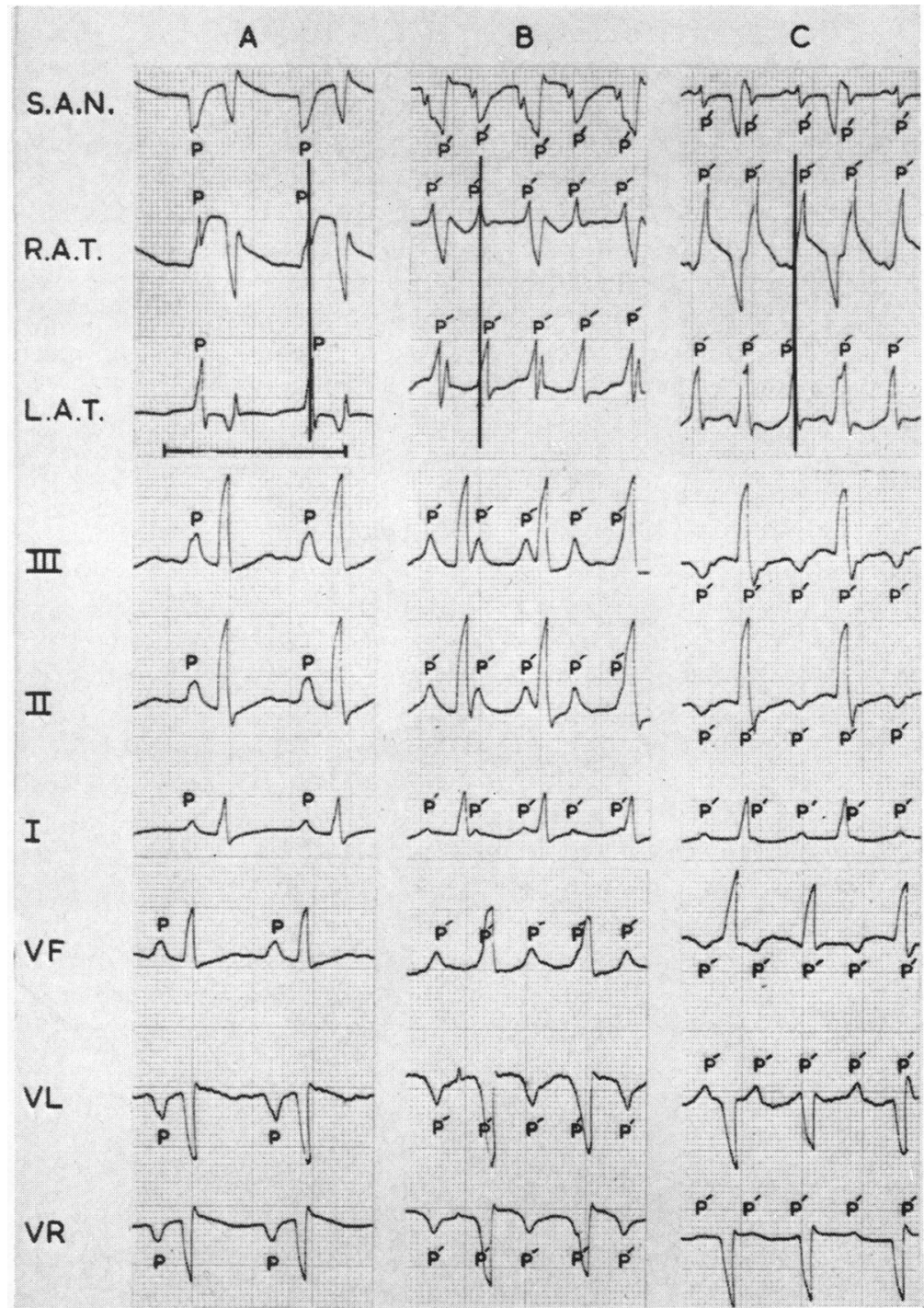

FIG. 1.-Reversal of the sequential activation process in direct atrial and limb leads in the same animal. S.A.N. = direct lead from sinus node. R.A.T. = direct lead from right atrial appendage. L.A.T.=direct lead from left atrial appendage. $\mathbf{P}=$ sinus beats. $\mathbf{P}^{\prime}=$ Flutter beats.

(A) Normal sinus rhythm. The thick vertical line shows that the two atria are beating in phase. $\mathbf{P}$ waves are positive in bipolar limb leads and in VF, and negative in VR and VL.

(B) After the production of atrial flutter. The thick vertical line shows that the activation of the right atrial appendage now precedes that of the left atrium by $0.024 \mathrm{sec}$. Limb leads show a change in the amplitude and form of flutter beats but their direction remains the same as that of sinus beats in excerpt A. The flutter rate is 450 a minute.

(C) The flutter was terminated by brief electrical stimulation and was reinduced 5 minutes later by electrical stimulation. The flutter rate is 452 a minute and the reversal phenomenon has occurred. The thick vertical line shows that the activation of the right atrial appendage now follows that of the left atrial appendage by precisely $0.024 \mathrm{sec}$. (reverse of excerpt B). Flutter waves are now positive in leads I, VR, and VL and negative in leads II, III, and VF.

The configuration of the QRS complexes remained unchanged in all the three excerpts. Time scale $=1 \mathrm{sec}$. 

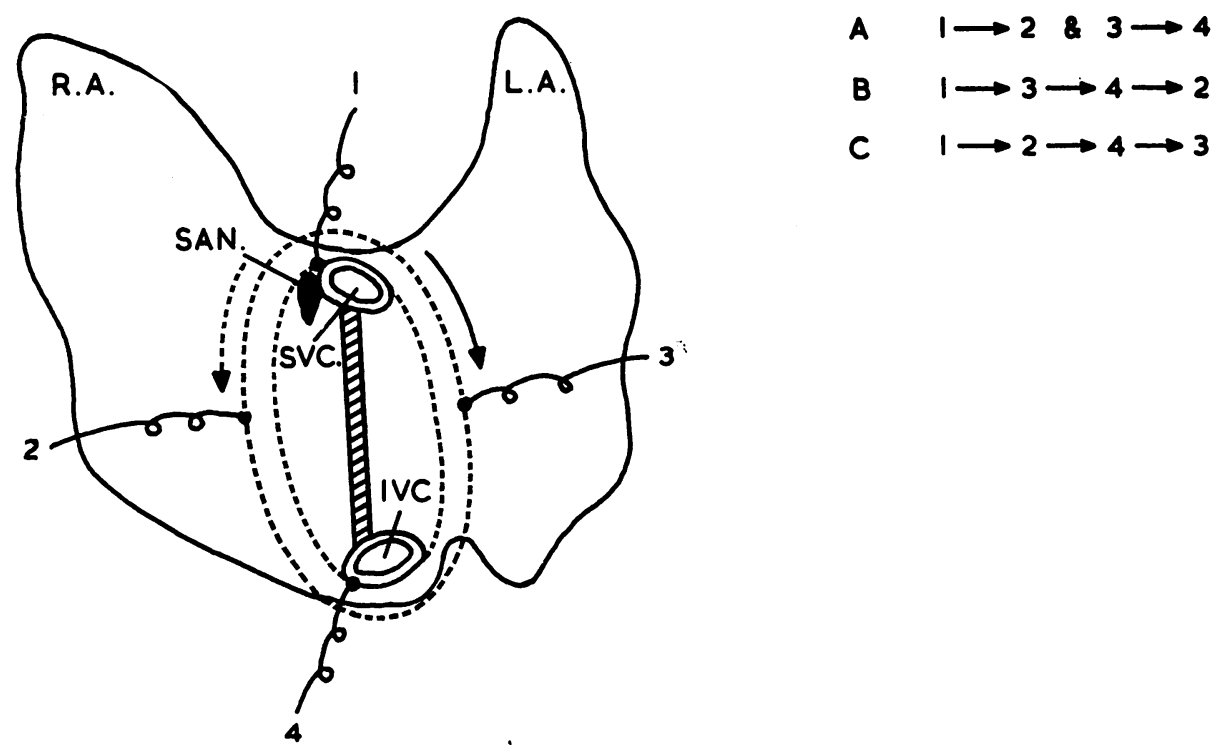

A
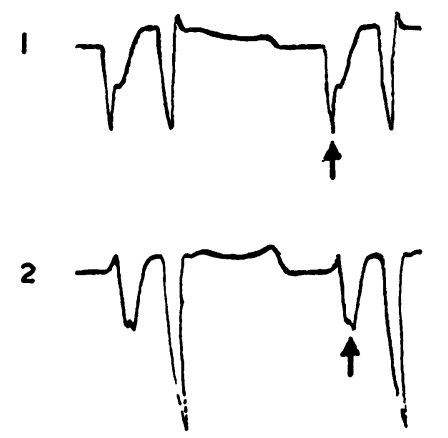

3
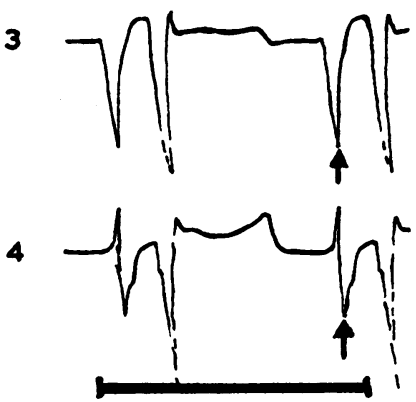

B

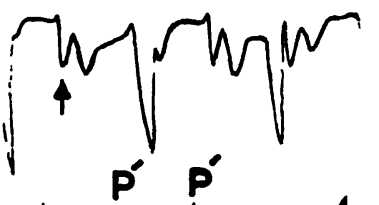

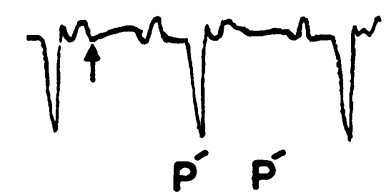

C

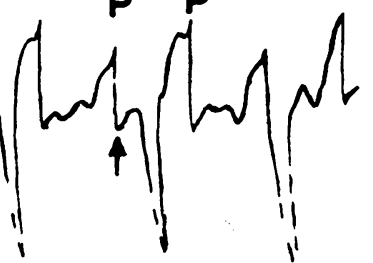

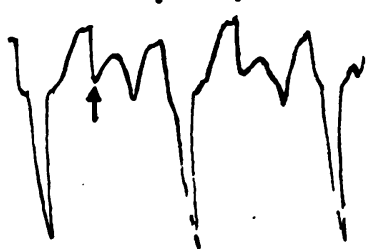

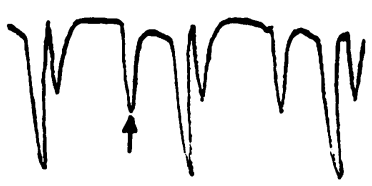

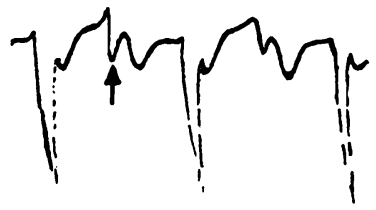

FIG. 2.-Time course of the excitation process around the hypothetical circus pathway in atrial flutter. Direct electrograms recorded from four points on the circus pathway as shown in the diagram. ${ }^{*}$ The intrinsic deflections of a single flutter beat are marked with arrows in each excerpt. Electrode No. 1 was used as the reference point.

(A) Sinus rhythm. The impulse spread is radial from the region of sinus node. The intrinsic deflections are described simultaneously at electrodes 2 and 3 , and at electrode 4 last of all.

(B) During first episode of atrial flutter, the impulse spread is unidirectional in a clockwise direction $(1 \rightarrow 3 \rightarrow 4$ $\rightarrow 2$ ). Atrial rate is 448 a minute.

(C) During second episode of flutter in the same animal. The impulse spread is now anticlockwise and is unidirectional $(1 \rightarrow 2 \rightarrow 4 \rightarrow 3)$, this is reverse of excerpt B. Atrial rate is 448 a minute. The atrial cycle $\left(\mathrm{P}^{\prime}-\mathrm{P}^{\prime}\right.$ interval) at a flutter rate of $448 \mathrm{a}$ minute is $0 \cdot 134 \mathrm{sec}$. The time taken by the impulse to travel from electrodes 1 to 3,3 to 4,4 to 2 , and 2 to 1 in excerpt $B$ is $0.033 \mathrm{sec}$. in each case. The total circuit time calculated by adding these four segmental time intervals is $0.132 \mathrm{sec}$., and this is almost equal to the atrial cycle $\left(\mathbf{P}^{\prime}-\mathbf{P}^{\prime}\right.$ interval). Thus the atrial cycle in flutter equals the time taken by the impulse to complete one circuit around the circus pathway. The same segmental measurements also apply to excerpt $\mathbf{C}$, in the reverse direction. Time scale $=0.5 \mathrm{sec}$.

* To place the electrode No. 3, the right atrium was lifted from its bed and a cotton tipped electrode was pushed under it parallel to the superior vena cava to a point opposite to electrode No. 2. 
A.

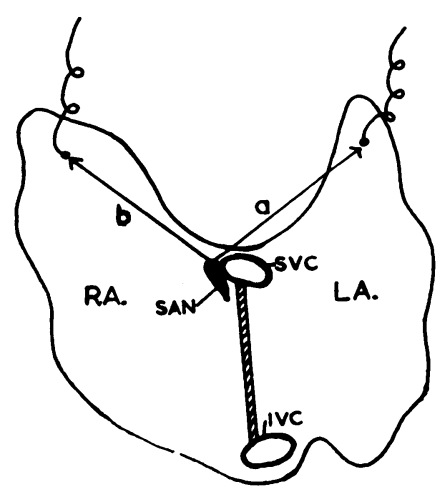

B.

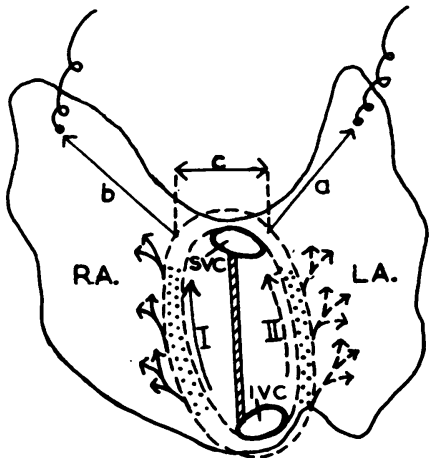

C.

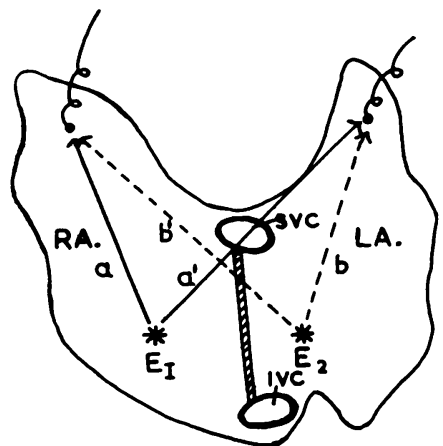

FIG. 3.-Diagrammatic illustration of sequential activation process depicted electrocardiographically in Fig. 1 . R.A.=right atrium; L.A. =left atrium; S.V.C. = superior vena cava; I.V.C. $=$ inferior vena cava; S.A.N. =sinoatrial node.

(A) Normal sinus rhythm. The electrodes at the two atrial appendages are so placed that sinus impulses (intrinsic deflections) reach them at the same time.

(B) Explanation of reversal process on the basis of the circus movement theory. Arrow I. The impulse circulates in a clockwise direction and reaches the right atrial appendage $0.024 \mathrm{sec}$. earlier than the left (Fig. 1 excerpt B), because to reach the left atrium the impulse travels an additional segment of the circuit labelled "c". Arrow II. The reversal has occurred (Fig. 1, excerpt C). The impulse circulates in an anticlockwise direction and reaches the right atrial appendage $0.024 \mathrm{sec}$. after the left. The fixed time lag of $0.024 \mathrm{sec}$. is because the impulse travels the same additional segment of the circuit (labelled c) to reach the right atrium.

(C) Explanation of the reversal process on the basis of ectopic focus theory. Suppose that E1 is the site of ectopic focus, the impulse reaches the right atrium earlier than the left because the focus is situated nearer to this electrode, i.e. $a<a^{\prime}$. After reversal the impulse reaches the left atrium earlier than the right atrium. To explain this it must be postulated that $b<b^{\prime}$ and the focus is somewhere in the left atrium, yet the electrical stimulation is always applied to the right atrium at the same spot. Further to explain the fixed time lag of $0.024 \mathrm{sec}$. it must be presumed that the ectopic focus develops in a definite area $E 2$, such that $a=b$ and $a^{\prime}=b^{\prime}$. The reversal process is therefore more readily explained on the basis of circus movement theory than on the ectopic focus theory.

unidirectional conduction of impulses in a clockwise or anticlockwise direction (Fig. 2). The observation that the flutter cycle $\left(\mathbf{P}^{\prime}-\mathbf{P}^{\prime}\right.$ interval) is equal to the time taken by the impulse to complete one circuit, also supports the circus movement theory. The sequential activation of the atria and its reversal in consecutive episodes of flutter is also easier to explain on the basis of the theory (Fig. 3). To explain the phenomenon of sequential activation on the basis of the ectopic focus theory, it must be postulated that the ectopic focus sometimes develops in the right atrium and at others in the left atrium, though the electrical stimulation is always applied to the same area of the right atrium. Further, to account for the fixed time lag of sequential activation, before and after the reversal has taken place, an unlikely assumption must be made that the ectopic focus develops in two identically situated areas of both the atria (Fig. 3, sites E1 and E2). Again, such an assumption will not explain the unidirectional spread of impulses.

The two distinct patterns of flutter waves observed in experimental flutter (Fig. 1) are also encountered in limb lead records of atrial flutter in man published by other workers (Barker, 1952; Goldberger, 1952; Lipman and Massie, 1959). This suggests that there is a similarity between experimental and clinical atrial flutter. It is, therefore, suggested that the limb lead records of those clinical cases that exhibit repeated attacks of atrial flutter should be studied for the reversal of flutter waves. If one pattern of flutter waves changes into the other in a subsequent episode of flutter in the same patient, it would be convincing evidence in support of the circus movement theory in man.

It has been demonstrated that once cholinergic influences convert atrial flutter into fibrillation, the arrhythmia terminates abruptly in most of the experiments. This is due to a breaking up of the circus movement brought about by excessive shortening of the refractory period, as suggested by 
Lewis, Drury, and Bulger (1921a): as the cholinergic influence disappears sinus rhythm returns. Clinical atrial flutter responds to digitalis therapy in a similar fashion. Digitalis by virtue of its reflex vagomimetic action also converts flutter into fibrillation, and when the drug is withdrawn several cases revert to sinus rhythm (Goodman and Gilman, 1955): On the basis of these similarities in experimental and clinical flutter, it is suggested that intravenous acetylcholine may prove useful in the treatment of clinical atrial flutter. Schoolman et al. (1960) tried acetylcholine in 14 cases of atrial flutter in man, but the results were not encouraging and only two cases reverted to sinus rhythm. These workers, however, did not mention whether the dose of acetylcholine used was successful in converting flutter into fibrillation or not. It is important that the dose of acetylcholine used should be large enough to convert flutter into fibrillation, i.e. the circus movement must be broken up to enable the sinus node to resume control of the cardiac rhythm.

\section{SUMMARY}

The mechanism of post-stimulatory atrial flutter in the anæsthetized dog was investigated by employing direct atrial and limb leads. By timing the impulse propagation along the hypothetical circus pathway unidirectional spread of the impulse in a clockwise or anticlockwise direction has been demonstrated. The direction can be reversed in a subsequent episode of flutter in the same animal.

Atrial appendages show sequential activation in flutter, and the time lag remains unchanged when the reversal phenomenon occurs in a subsequent episode in the same animal. Two distinct patterns of flutter waves are seen in the limb lead records, depending upon whether the activation of right atrium precedes or follows that of the left atrium. These two patterns of flutter waves also occur in the limb lead records of clinical atrial flutter, and it is suggested that electrocardiographic records of patients exhibiting repeated attacks of flutter should be studied for the reversal of flutter waves.

The similarities of experimental and clinical flutter are discussed and it is suggested that intravenous acetylcholine may prove useful in the treatment of atrial flutter in man.

I am very grateful to Dr. H. C. Stewart for providing the facilities to carry out this work and for many useful suggestions. This work forms part of a thesis approved in the University of London for the award of the Ph.D. degree, 1962.

\section{REFERENCES}

Barker, J. M. (1952). The Unipolar Electrocardiogram, 1st ed., p. 523. Appleton-Century-Crofts, New York. Goldberger, E. (1952). Unipolar Lead Electrocardiography and Vectocardiography, 1st ed., p. 421. Kimpton, London.

Goodman, L. S., and Gilman, A. (1955). The Pharmacological Basis of Therapeutics, 2nd ed., p. $704 . \quad$ Macmillan, New York.

Lewis, T. (1920). Observations upon flutter and fibrillation. Part IV. Impure flutter; theory of circus movement. Heart, 7, 293.

(1925). The Mechanism and Graphic Registration of the Heart Beat, 3rd ed. Shaw and Sons, London.

- Drury, A. N., and Bulger, H. A. (1921a). Observations upon flutter and fibrillation. Part VII. The effects of vagal stimulation. Heart, 8, 141.

,$- \frac{}{2}$, and Iliescu, C. C. (1921b). A demonstration of circus movement in clinical flutter of the auricles. Heart, 8, 341 .

- Feil, H. S., and Stroud, W. D. (1920). Observation upon flutter and fibrillation. Part II. The nature of auricular flutter. Heart, 7, 191.

Lipman, B. S., and Massie, E. (1959). Clinical Scaler Electrocardiography, 4th ed., p. 411. Year Book Publishers, Chicago.

Prinzmetal, M., Corday, E., Oblath, R. W., Kruger, H. E., Brill, I. C., Fields, J., Kennamer, S. R., Osborne, J. A., Smith, L. A., Sellers, A. L., Flieg, W., and Finston, E. (1951). Auricular flutter. Amer. J. Med., 11, 410.

- Goldman, A., Gerlach, E., and Kennamer, R. (1953). Nature of spontaneous auricular flutter in man. $J$. Amer. med. Ass., 153, 553. 
Rosenblueth, A., and Garcia Ramos, J. (1947). Studies on flutter and fibrillation. II. The influence of artificial obstacles on experimental auricular flutter. Amer. Heart J., 33, 677.

Rothberger, C. J., and Winterberg, H. (1914). Über Vorhofflimmern und Vorhofflattern. Pfügers Arch. ges. Physiol., 160, 42.

Schoolman, H. M., Bernstein, L. M., Littman, A., and Pascale, L. R. (1960). Acetylcholine in differential diagnosis and treatment of paroxysmal tachycardia. Amer. Heart J., 60, 526. 